\title{
Bioanalytical reviews: foreword
}

\author{
Otto S. Wolfbeis • Frank-Michael Matysik
}

Published online: 27 November 2009

(C) Springer-Verlag 2009

Dear reader,

This is the first issue of Springer's Bioanalytical Reviews (BAR). The journal's mission is to cover progress in the strongly growing area of bioanalytical sciences. BAR will publish reviews only and will be a unique source of information for anybody using or developing bioanalytical methods in areas such as medicine, biology, biochemistry, genetics, pharmacology, biotechnology, pharmaceutical screening, bioinformatics, the various -omics, and the like.

As outlined in the "Aims and Scopes", BAR covers all kinds of methods of instrumental analysis and laboratory techniques. This first issue is quite representative. Adam Heller's review is concerned with the hot area of sensing nitrogen monoxide, one of the major messenger molecules in physiology. Sweedler et al. review methods for analyzing Damino acid peptides. Tsunoda covers bioanalytical options resulting from the hyphenation of separation techniques and chemiluminescence detection. Malik et al. review new trends in solid phase microextraction of (mainly) biomaterial. Robelek summarizes the progress made in surface plasmon resonance (an important label-free method sensing scheme) in the context of cellular interactions and cellular reactions to extra- and intracellular stimuli, while Moises and Schaeferling report on new developments in the field of biosensor arrays for food toxins in milk.

O. S. Wolfbeis $(\bowtie) \cdot$ F.-M. Matysik $(\bowtie)$

Institute of Analytical Chemistry, Chemo- and Biosensors,

University of Regensburg,

Regensburg 93040, Germany

e-mail: otto.wolfbeis@chemie.uni-r.de

e-mail: Frank-Michael.Matysik@chemie.uni-r.de
The articles clearly reveal the width of areas to be covered by reviews published in BAR. We like to encourage prospective authors of a review article for BAR to contact one of the editors or the editorial board.

Regensburg, 17 November 2009

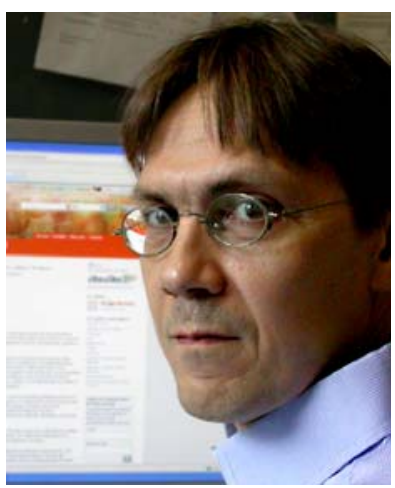

Frank-Michael Matysik

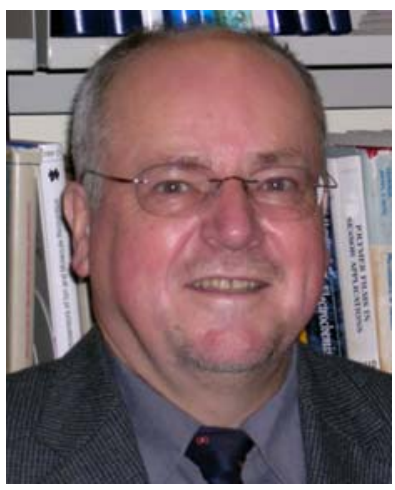

Otto S. Wolfbeis 